\title{
The Effects of Weber Numbers on Central Collision Between Two Droplets
}

\author{
Feng Wei \\ Department of Space Equipment \\ Equipment Academy \\ Beijing, CHINA \\ e-mail: fengwei_85@163.com \\ Nie Wansheng \\ Department of Space Equipment \\ Equipment Academy \\ Beijing, CHINA \\ e-mail: nws1969@126.com
}

\author{
Zheng Zhi \\ Department of Space Equipment \\ Equipment Academy \\ Beijing, CHINA \\ e-mail: 532560921@qq.com \\ Chen Feng \\ Department of Space Equipment \\ Equipment Academy \\ Beijing, CHINA \\ e-mail: cfel@qq.com
}

\begin{abstract}
The main objectives of the present article are in simulating the collision phenomenon between two liquid droplets and in discussing the influence of Weber numbers on droplets collision. The investigation is completed based on the finite volume numerical solution of the Navier-Stokes equations, in the formulation, expressing the flow field of the two phases, liquid and gas, coupled with the volume of fluid (VOF) method for tracking the liquid-gas interface. And three-dimensional simulations are used to investigate the mechanisms of coalescence and separation of the droplets at difference Weber numbers. The model and method are validated by comparison with available experimental data for the temporal movement of the impinging droplet. Results show that, numerical simulations provide realistic droplet collision behaviors. The droplets coalescence, deformation and break up processes after impinging are investigated at low and high Weber numbers, and the outcomes of a collision show the difference between various Weber numbers.
\end{abstract}

Keywords- droplet collisions; volume of fluid method; interface tracking; break up; surface tension

\section{INTRODUCTION}

As it is known that droplet breakup is induced by interactions between spray droplets and gas motion mainly, which is called aerodynamic breakup, and droplet collision is a mutual impact caused by differences in the velocity and direction of the droplets [1]. Droplet collisions are very important in liquid rocket engine, especially in the case of a dense and high Weber number region near injector faceplate. As there are hundreds of injectors in the liquid rocket engine, droplet collision is expected to be a repetitive events, which resulting in breakups that form several satellite droplets or a combination of droplet masses, and that can significantly affect the vaporization and combustion characteristics [2]. Therefore, the collision dynamics of liquid droplets and the fundamental mechanism of the simplest form of droplet collision have been of interest in recent years.

Numerous earlier experimental studies [3, 4] have been mainly focused on the basic phenomena of binary droplet collision, with emphasis on identifying and interpreting distinct collision outcomes and their dependence on the collision parameters, which can help us in a better understanding of droplet collision mechanisms. The researchers have found that possible droplets collision outcomes from observing experimental results can exhibit five distinct collision regimes, namely: (I) coalescence following minor deformation, (II) bouncing, (III) coalescence following substantial deformation, (IV) coalescence followed by separation for near head-on collisions, and (V) coalescence followed by separation for off-center collisions [5, 6]. However, due to the quite large number of parameters involved in the process, it appears more and more difficult to control the important parameters accurately in experiment, especially the Weber number is high. As the CFD technologies development, numerical simulation studies on two-phase flow thus remain a research topic of high interest and many different methods have been developed to investigate the atomization and droplet transport, such as the particle-incell (PIC) method [7], the smoothed particle hydrodynamics (SPH), the volume of fluid (VOF) [8], the level set method [9], the moving particle semi-implicit (MPS) method [10], and the Lattice Boltzmann method. There numerical studies have been carried out using either "front tracking" methods or "front capturing" methods. Front tracking methods are based on the Lagrangian tracking and marked particles to attach the interface motion. These methods are suitable for the problem that the irregularities of interface curvature are not too large. But they are not suitable to adapt to describing topological changes of the interface. The Volume of Fluid method (VOF) and the level set method are the main approaches are involved in the "front capturing" method. The VOF method describes the volumetric fraction of each phase in grid cells. They were developed in the 1980s and have been commonly used for some years. It is a very simple and efficiency method, and has been used in many problems of two phase problem. Recently interface reconstruction can be avoided in VOF method by assuming a continuous volumetric fraction throughout the whole computational domain, which means that an interface thickness is introduced. 
Nikolopoulos et al. [11] using VOF methodology coupled with an adaptive local grid refinement technique to investigate the effects of liquid or gas properties and droplet size ratio on the collision outcome between two unequal-size droplets for the reflexive regime. Theodorakakos et al. [12] using VOF method to investigate the problem that droplet impinging onto a wall film, in the simulation they found that three-dimensional numerical simulations proved to be accurate at acceptable levels, predicting not only the evolution of the formation of the lamella during the initial stages of impact but also the 3-D formation of satellite droplets realized with high impact Weber numbers. As it is seen, that in most of numerical simulations they focused their interest on the coalescence process and also ignore the collision induced breakup processes from the assumption that all droplets inside spray move in the same direction, because relative velocities and Weber numbers are not enough to produce the breakup.

The main aim of present work is to investigate the head-on binary droplet collisions for various Weber numbers (including low and high Weber numbers) numerically. We firstly present the volume of fluid method which was adopted for the tracking of the liquid-gas interface and its coupling with incompressible NavierStokes equations. The reliability of the methodology is first established by comparing the numerical results to experimental data and then three-dimensional simulations are conducted to investigate the effects of Weber numbers on outcomes of collision.

\section{THE MATHEMATICAL MODELS}

\section{A. Determination of Important Parameters}

In general, the binary collision between two droplets are mainly governed by the diameter $D$ of the droplet, surface tension $\sigma$, liquid density $\rho_{l}$, and droplets corresponding initial impact velocities $\mathbf{U}_{12}$. Many experimental and simulation investigations have proved that, regardless of the liquid type, the outcomes of collision process can be delineated by three important dimensionless parameters [13]: the Weber number (We), droplet diameter ratio $(\Delta)$ and impact parameter $(B)$, which are, respectively, defined by

$$
\begin{gathered}
W \mathrm{e}=\frac{\rho_{l} D_{2}\left|\mathbf{U}_{12}\right|^{2}}{\sigma} \\
\Delta=\frac{D_{2}}{D_{1}} \\
B=\frac{2 X}{D_{1}+D_{2}}
\end{gathered}
$$

where the subscripts 1 and 2 indicate the large and small droplet, respectively. The impact parameter $B$ characterizes the eccentricity of the collision, and $X$ as shown in Fig . 1 is defined as the distance from the center of one droplet to the relative velocity vector placed on the center of the other droplet.

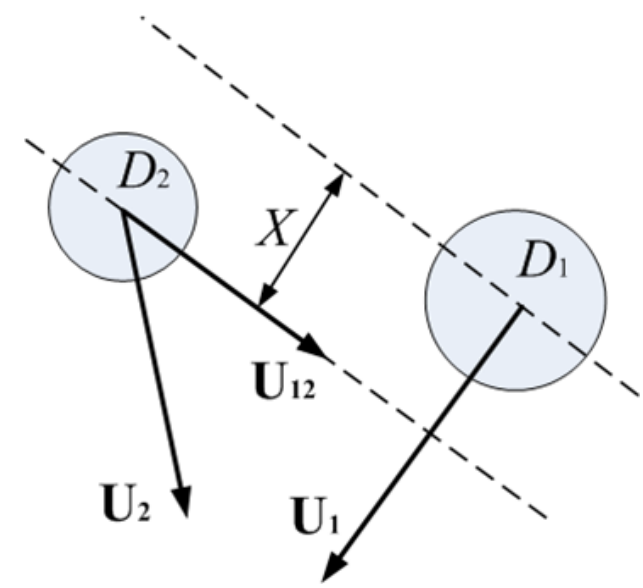

Figure 1. Schematic of the binary droplet collision

\section{B. The Equation of Flow}

The multiphase flow is considered laminar and incompressible, and the unsteady Navier-Stokes equations and continuity equation are solved for both gas and liquid phase in a unified computation domain, and therefore the continuity and momentum equations for the fluid can be written, respectively, as follows:

$$
\begin{gathered}
\frac{\partial \rho}{\partial t}+\nabla \bullet(\rho \mathbf{U})=0 \\
\frac{\partial(\rho \mathbf{U})}{\partial t}+\nabla \bullet(\rho \mathbf{U U})=-\nabla p+\nabla \bullet(\mu \nabla \mathbf{U})+\mathbf{F}
\end{gathered}
$$

Where $\rho, \mathbf{U}, \mu$ and $p$ are the density, velocity, dynamic viscosity and pressure. The source term $\mathbf{F}$ representing the liquid surface tension force is expressed as:

$$
\mathbf{F}=\int_{S(t)} \sigma \kappa \mathbf{n} \delta \mathrm{d} S
$$

Where $\delta$ is Dirac function, $\kappa$ and $\mathbf{n}$ are curvature and unit normal vector the surface, respectively.

\section{VOF Method for Interface Tracking}

A volume fraction (VOF) indicator function $\alpha$ is introduced in the unified computation domain. The $\alpha$ function is equal to 1 for a point of the computational domain inside the liquid phase, 0 inside the gas phase and takes values between 0 and 1 in the gas-liquid interface. The transport equation for the VOF indicator $\alpha$ is written as:

$$
\frac{\partial \alpha}{\partial t}+\nabla \bullet(\mathbf{U} \alpha)=0
$$

The values of density and dynamic viscosity within the flow field are then calculated using linear interpolation between the values of the two phases weighted with the volume fraction $\alpha$ :

$$
\begin{aligned}
& \rho=\alpha \rho_{l}+(1-\alpha) \rho_{g} \\
& \mu=\alpha \mu_{l}+(1-\alpha) \mu_{g}
\end{aligned}
$$

\section{The Nmerical Slution Pocedure}

The equations are discretized by the finite volume method. Convection terms and diffusion terms are discretized by a second order accuracy upwind scheme and central difference scheme respectively. The backward 
scheme is used to discretize the time terms. The coupling between pressure and velocity is solved by PISO algorithm. The details process for solving the equations as shown in Fig .2. At the beginning of the solution, the VOF function must be initialized through the initial and boundary conditions, and then based on the PISO algorithm to solve the N-S equations and the pressure correction equation; after updating the fields of velocity and pressure, the transport function of volume fraction can be solved and the interface between gas and liquid will be reconstructed, To solve the corresponding equations circularly until it reaches the convergence condition.

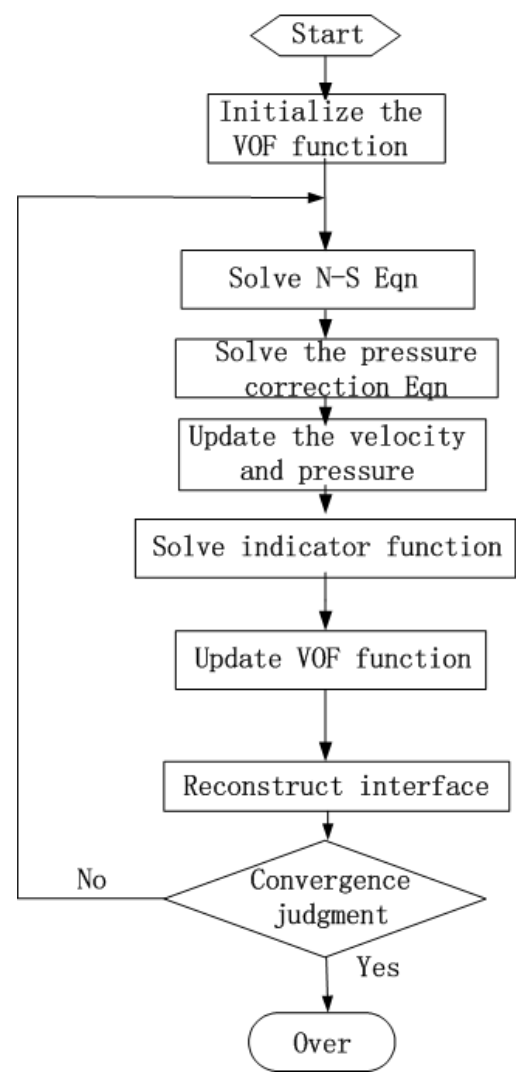

Figure 2. Schematic diagram of the solving process

\section{RESULTS AND DISCUSSIONS}

\section{A. Comparison with Experiment}

In all simulations, droplet diameter ratio $(\Delta)$ and impact parameter $(B)$ are equal to 1 and 0 , respectively. The diameters of droplets are fixed at $0.8 \mathrm{~mm}$, and the droplet relative velocity is imposed to fit the given Weber number. The grid size is $80 \times 100 \times 100$ in the $3 \mathrm{D}$ computation domain. The physical properties of gas and liquid are given in Table 1.

TABLE I. PHYSICAL PROPERTIES OF BOTH PHASE

\begin{tabular}{|c|c|c|c|}
\hline & $\sigma\left(\mathrm{kg} \mathrm{s}^{-2}\right)$ & $\rho_{\left(\mathrm{kg} \mathrm{m}^{-3}\right)}$ & $\mu_{\left(\mathrm{kg} \mathrm{m}^{-1} \boldsymbol{s}^{-1}\right)}$ \\
\hline Air & - & 1.226 & $1.78 \times 10^{-5}$ \\
\hline Water & 0.072 & 1000 & 0.001137 \\
\hline
\end{tabular}

To verify the present numerical method, the head-on collision of two water droplets was simulated, and the Weber number, impact parameter and diameter are 23,0 and $0.8 \mathrm{~mm}$, respectively, as the experiment used [14]. A direct comparison for the evolution of droplet contours is shown in Fig .3. It can be seen that the numerical simulation results presented are in good agreement with experimental observations on water droplets movement, and the liquid phase behavior during collision process is qualitatively similar in both image sequences. So the model and methods can be used for the droplet collision study. From the Fig .3, it is also observed that at the initial time of collision, the coalescence of the two droplets is happen and the resulting single drop then stretches into a ring shape with a thin membrane. Under surface tension forces, the ring is then attracted to its center and the internal motion of the liquid leads to a cylinder. Due to the fairly high initial kinetic energy of the droplets, the cylinder is then lengthens until break-up occurs.

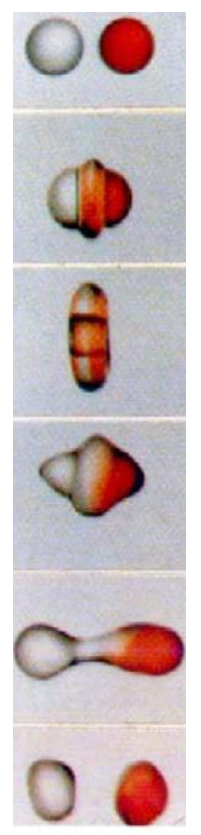

(a) Experimental results

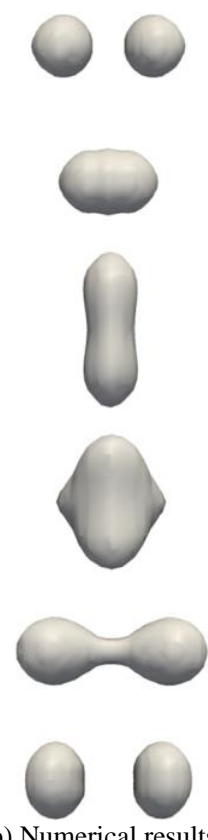

(b) Numerical results
Figure 3. Comparison between the experimental and numerical results with $W e=23$

\section{B. Low Weber Number}

Fig .4 presents the temporal evolution of the collision droplets shape at a low Weber number, which is equal to 10. We observe the coalescence of the two droplets and the resulting drop then starts to oscillate slightly but the momentum energy is too low to produce any break-up in the process. It can be seen that the oscillations of the resulting droplet are much small. As expected, the drop behavior is driven by surface tension forces. Due to the high curvature at impact, the drop is elongated in the perpendicular direction of collision and when it reaches its maximum elongation, the surface tension tends to draw back the droplet to its more stable shape and oscillations thus occur around the spherical shape. However, the initial kinetic energy of the droplets is still not enough to induce breakup of the cylinder shape observed on the drawing. After several times oscillation, droplet kinetic energy is reduced to 0 by viscous consumption. 


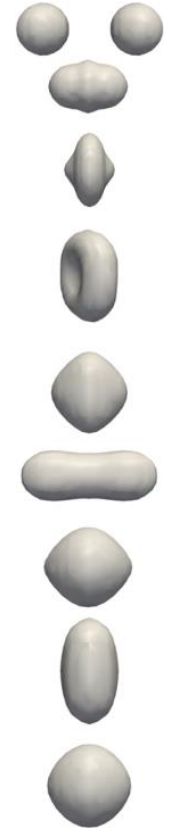

Figure 4. Coalescence at Low Weber Number

\section{High Weber Number}

In order to investigate the outcomes of droplets collision at high Weber numbers, a series of simulations were conducted with only the droplet relative velocity is increased while keeping the other parameters unchanged. While the Weber number is 100, 300 and 500, the relative velocity is thus equal to $3 \mathrm{~m} \mathrm{~s}^{-1}, 5.2 \mathrm{~m} \mathrm{~s}^{-1}$ and $6.7 \mathrm{~m} \mathrm{~s}^{-1}$, respectively. Simulation results are shown in Fig .5. The collision leads to coalescence firstly, and following by separation with the formation of many satellites, in Fig .5a. While the Weber number increasing higher, the result of collision droplet formed a very thinner and wider liquid sheet, and then uniform liquid ligaments appeared, finally the ligaments seperated into smaller droplets, as seen in Fig .5b and Fig .5c.

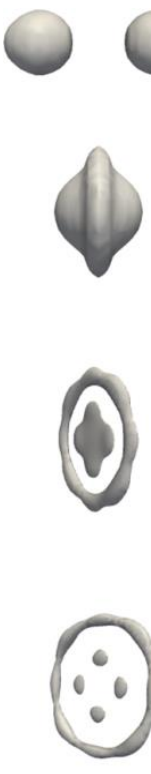

(a) $W e=100$
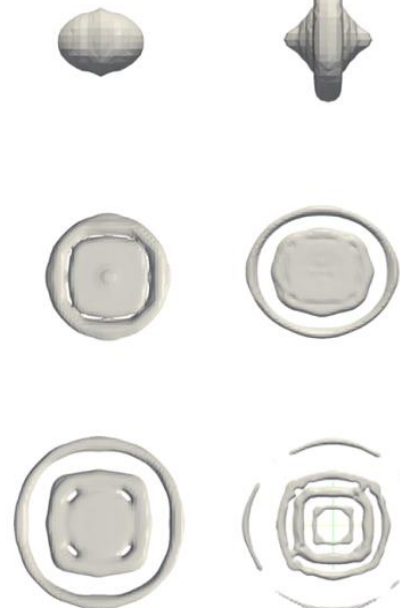

(b) $W e=300$

(c) $W e=500$
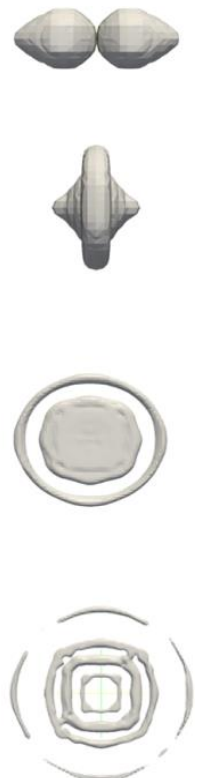

Figure 5. Effect of Weber numbers on the droplet collision

\section{CONCLUSIONS}

In the present work, simulations of binary droplet collision are conducted using the volume of fluid (VOF) method to track the liquid-gas interface. With threedimensional model, the investigation results show important details of the droplets collision, including droplet deformation, liquid sheet break up and smaller droplets producing processes in the impact region. For head-on collision of droplets, the stretching separation is the main breakup way at low Weber number, and while the Weber number is very large, collision droplets form a very thin liquid sheet firstly, and then the sheet breaks up at its outer region, and ligaments and drops disperse.

\section{ACKNOWLEDGMENT}

The financial support from the National Natural Science Foundation of China (91441123) is acknowledged.

\section{REFERENCE}

[1] S. Kim, D. J. Lee, C. S. Lee, "Modeling of Binary Droplet Collisions for Application to Inter-impingement Sprays,' International Journal of Multiphase Flow, vol, 35: 2009, pp. 533549, doi:10.1016/j.ijmultiphaseflow.2009.02.010.

[2] D. Liu, P. Zhang, C. K. Law, et al, "Collision Dynamics and Mixing of Unequal-size Droplets," International Journal of Heat and Mass Transfer, vol. 57, 2013, pp. 421-428.

[3] N. Ashgriz, P. Givi, "Coalescence Efficiencies of Fuel Droplets in Binary Collisions," Heat Mass Transfer, 1989, pp. 16:11

[4] N. Ashgriz, J.Y. Poo, "Coalescence and Separation in Binary Collision of Liquid Drops,” J. Fluid. Mech. vol. 221, 1990, pp. 183-204.

[5] A. Munnannur, R. D. Reitz, "A New Predictive Model for Fragmenting and Non-fragmenting Binary Droplet Collisions,' International Journal of Multiphase Flow, vol. 33, 2007, pp. 873896, doi:10.1016/j.ijmultiphaseflow.2007.03.003.

[6] G. H. Ko, H. S. Ryou, "Modeling of Droplet Collision-induced Breakup Process," International Journal of Multiphase Flow, vol. 31, 2005, pp. 723-738, doi:10.1016/j.ijmultiphaseflow.2005.02.004.

[7] A.A. Amsden, "The Particle-in-cell Method for the Calculation of the Dynamics of Compressible Fluids," Los Alamos Scientific Laboratory Report LA, 1966, 3466.

[8] C. W. Hirt, B. D. Nichols, "Volume of Fluid(VOF) Method for the Dyanmics of Free Boundaries," Journal of Computational Physics, vol. 39, 1981, pp. 201-225.

[9] S. Tanguy, A. Berlemont, "Application of a Level Set Method for Simulation of Droplet Collisions," International Journal of Multiphase Flow, vol. 31, 2005, pp. 1015-1035, doi:10.1016/j.ijmultiphaseflow.2005.05.010.

[10] Z.G Sun, G. Xi, X. Chen, "Numerical Simulation of Binary Collisions Using a Modified Surface Tension Model with Particle Method," Nuclear Engineering and Design, vol. 239, 2009, pp. 619-627, doi:10.1016/j.nucengdes.2008.12.017.

[11] N. Nikolopoulos, G. Bergeles, "The effect of gas and liquid properties and droplet size ratio on the central collision between two unequal-size droplets in the reflexive regime," International Journal of Heat and Mass Transfer, vol. 54, 2011, pp. 678-691, doi:10.1016/j.ijheatmasstransfer.2010.09.002.

[12] N. Nikolopoulos, A. Theodorakakos, G. Bergeles, "Threedimensional Mumerical Investigation of a Droplet Impinging Normally onto a Wall Film," Journal of Computational Physics, vol. 225, 2007, pp. 322-341, doi: 10.1016/j.jcp.2006.12.002.

[13] M. Orme, "Experiments on Droplet Collisions, Bounce, Coalescence and Disruption," Energy Combust. Sci. vol. 23, 1997, pp. 65-79.

[14] N. Ashgriz, J.Y. Poo, "Coalescence and Separation in Binary Collisions of Liquid Drops,” J. Fluid Mech, vol. 221, 1990, pp. 183-204. 